\title{
Oltipraz ameliorates the progression of steatohepatitis in Nrf2-null mice fed a high-fat diet
}

\author{
Toshinori Kamisako* and Yuji Tanaka \\ Department of Clinical Laboratory Medicine, Kindai University Hospital, 377-2 Ohnohigashi, Osakasayama, Osaka 589-8511, Japan
}

(Received 13 May, 2021; Accepted 3 August, 2021)

\begin{abstract}
Oltipraz, a synthetic dithiolethione, has chemopreventive effect through nuclear factor erythroid 2-related factor 2 (Nrf2) activation. Nrf2 is known to be involved in the development of experimental steatohepatitis in rodents. In this study, to evaluate the effect of oltipraz on lipid and bile acid metabolism, wild-type and Nrf2-null mice were fed the standard diet (containing $4 \%$ soybean oil) with or without oltipraz. Based on these results, we examined the effect of oltipraz on the experimental steatohepatitis in high-fat diet (containing $4 \%$ soybean oil and $20 \%$ lard) fed Nrf2-null mice. Oltipraz induced hepatic mRNA expression of peroxisome proliferator-activated receptor $\alpha$, carnitine palmityl transferase 1, and bile salt export pump by Nrf2 independent mechanisms. In Nrf2-null mice fed a high-fat diet for 12 weeks, moderate to severe inflammation and fibrosis were observed. Oral administration of oltipraz suppressed the degree of inflammation and fibrosis in Nrf2-null mouse liver fed a high-fat diet. These histopathological findings approximately corresponded to the data of mRNA expression of tumor necrosis factor $\alpha$, monocyte chemoattractant protein-1, Timp-1, and collagen type 1a1. These results indicated that oltipraz administration ameliorated liver injury by Nrf2 independent manner in a model of steatohepatitis generated by Nrf2-null mice with high-fat diet.
\end{abstract}

Key Words: nuclear factor erythroid 2-related factor 2, oltipraz, high-fat diet, steatohepatitis, Nrf2-null mice

$\mathrm{O}$ ltipraz (OPZ) [4-methyl-5-(2-pyrazinyl)-1,2-dithiol-3-thione], a synthetic dithiolethione, is known as a chemopreventive agent. The chemopreventive effect of OPZ is related to the enhancement of binding activity between nuclear factor erythroid 2-related factor 2 (Nrf2) and antioxidant response element (ARE). At present OPZ is known as a potent Nrf2 activator. The transcription factor Nrf2 is a crucial molecule in the maintenance of redox homeostasis. Nrf2 induces the expression of antioxidant and detoxifying enzymes, glutathione homeostasis, and transport of xenobiotics, including glutathione $S$-transferase A1 (Gsta1), $\mathrm{NAD}(\mathrm{P}) \mathrm{H}$ :quinone oxidoreductase 1 (Nqo1), and multidrug resistance associated proteins (Mrps) by binding to antioxidant responsive elements in the promoters of these genes. ${ }^{(1,2)} \mathrm{Nrf} 2$ has additional roles in lipid and bile acids metabolism beyond detoxification. With respect to the role of $\mathrm{Nrf} 2$ in lipid metabolism, previous studies demonstrated that Nrf2 inhibits lipid accumulation and lipid peroxidation in mouse liver after feeding a high-fat diet (HFD) by interfering with lipid-related pathways. Furthermore, enhanced expression of Nrf2 in Kelch-like ECH-associated protein 1 (Keap1) knockdown mice attenuates fatty liver produced by a methionine- and choline-deficient (MCD) diet. ${ }^{(3,4)}$ Concerning the role of Nrf2 in bile acids metabolism, Nrf2 protects against hepatotoxicity induced by an intraperitoneal injection of lithocholic acid. ${ }^{(5)} \mathrm{Nrf} 2$ modulates bile acid homeostasis in mouse liver, and Nrf2 regulates bile salt export pump (Bsep), multidrug resistance associated protein (Mrp) $4,{ }^{(6,7)}$ and cholesterol $7 \alpha$-hydroxylase (Cyp7a1) expression. ${ }^{(8)}$ As OPZ is a potent Nrf2 activator, it induces Nqo1 along with other enzymes and bile acid transporters, including Bsep, Mrp2, Mrp3, and Mrp4. ${ }^{(9,10)}$

Nrf2-null mice are known to develop steatohepatitis when fed a MCD diet or a high-fat diet. ${ }^{(11,12)}$ The effects of Nrf2 deletion on antioxidant defense mechanisms, bile acid metabolism, and lipid metabolism are involved in the development of experimental steatohepatitis in rodents. Several types of Nrf2 activators have been reported to improve liver injury in the experimental steatohepatitis in rodents. ${ }^{(13-15)}$ It has been reported that OPZ protects against alpha-naphthylisothiocyanate (ANIT)-induced hepatotoxicity and choline-deficient, L-amino acid defined diet (CDAA)induced liver injury. ${ }^{(16,17)}$ This hepatoprotective effect of OPZ is thought to be due to Nrf2 activation. However, OPZ also improves ANIT-induced hepatotoxicity in Nrf2-null mice, ${ }^{(16)}$ suggesting that OPZ may also have an Nrf2 independent hepatoprotective effect. Indeed, OPZ is also known to activate constitutive androstane receptor (CAR) in addition to Nrf2.(18) In the present study, we investigated whether OPZ has a protective effect against HFD-induced liver injury in Nrf2-null mice, and whether OPZ has Nrf2 independent hepatoprotective effect.

\section{Materials and Methods}

Materials. Milk casein, corn starch, $\alpha$-corn starch, mineral AIN-93G mixture and vitamin AIN-93VX mixture were purchased from Clea Japan (Osaka, Japan). Soybean oil and lard were purchased from Oriental Yeast (Tokyo, Japan) and Yamakei (Osaka, Japan), respectively. OPZ was purchased from LKT Laboratories, Inc. (St. Paul, MN) and all other chemicals were purchased from Wako Pure Chemical Industries (Osaka, Japan), unless noted.

Animals. A colony of wild-type and Nrf2-null mice, generated originally by Itoh et al., were backcrossed with $\mathrm{C} 57 \mathrm{BL} / 6$ mice for ten generations (Itoh $\mathrm{K}$ et al., 1997). All mice were housed in the same animal care facility controlling for temperature $\left(22 \pm 2^{\circ} \mathrm{C}\right)$, humidity $(55 \pm 5 \%)$, and light (lights on; 07:00 19:00 h).

Experiment 1. To examine the effect of OPZ on lipid and bile acid metabolism, 15-week-old male wild-type and Nrf2-null mice ( $n=6$ /group) were divided into two groups fed the following

*To whom correspondence should be addressed.

E-mail: kamisako@med.kindai.ac.jp 
Table 1. Composition of the experimental diets (weight \%)

\begin{tabular}{lcccc}
\hline Ingredients & Standard & Standard/OPZ & HFD & HFD/OPZ \\
\hline Soybean oil & 4 & 4 & 4 & 4 \\
Lard & - & - & 20 & 20 \\
Oltipraz & - & 0.0067 & - & 0.0124 \\
Milk casein & 14 & 14 & 14 & 14 \\
L-Cystein & 0.18 & 0.18 & 0.18 & 0.18 \\
AIN-93G mineral mixture* & 3.5 & 3.5 & 3.5 & 3.5 \\
AIN-93VX vitamine mixture* & 1 & 1 & 1 & 1 \\
Cellulose powder & 5 & 5 & 5 & 5 \\
Corn starch & 46.57 & 46.57 & 26.57 & 26.57 \\
$\alpha-C o r n$ starch & 15.5 & 15.5 & 15.5 & 15.5 \\
Sucrose & 10 & 10 & 10 & 10 \\
Choline bitartrate & 0.25 & 0.25 & 0.25 & 0.25 \\
\hline
\end{tabular}

*The amount recommended for use is given by the American Institute of Nutrition. HFD, high fat diet; OPZ, oltipraz.

diets: 1) a standard diet (AIN-93, containing 4\% soybean oil) for 4 weeks (Standard group), 2) a standard diet containing OPZ (average intake of OPZ $9.3 \mathrm{mg} / \mathrm{kg} /$ day) for 4 weeks (Standard/ OPZ group).

Experiment 2. Seven-week-old male wild-type and Nrf2-null mice ( $n=6$ /group) were divided into two groups fed the following diets: 1 ) a high fat diet (HFD) (containing 4\% soybean oil and 20\% lard) for 12 weeks (HFD group), 2) a HFD for 8 weeks followed by a HFD containing OPZ (average intake of OPZ $8.6 \mathrm{mg} / \mathrm{kg}$ /day) for 4 weeks (HFD/OPZ group).

Diets. The composition of the experimental diets is shown in Table 1. As OPZ was fine powder, OPZ was thoroughly mixed with other compositions of each diet using a food mixer to obtain the desired concentrations in the feed. The dose and the duration of OPZ administration was chosen with reference to previous reports. ${ }^{(19,20)}$ Individual body weights and food intake were recorded once or twice a week, respectively. Based on these data, the amount of OPZ mixed in diets was adjusted each time to administer approximately same total amount of OPZ between groups when diets were prepared. Food was removed two hours prior to collecting tissues and blood samples were collected by heart puncture after anesthesia, and livers were harvested and stored at $-80^{\circ} \mathrm{C}$ until use. Animals received humane care as outlined in the Guide for the Care and Use of Laboratory Animals (NIH publication 86-23, revised 1985). Studies were approved by Kindai University Faculty of Medicine Animal Care and Use Committee.

Quantification of serum biochemical markers. Serum biochemical markers were quantified using biochemistry autoanalyzer Labospect 008 (Hitachi High-Technologies Corporation, Ibaragi, Japan). Serum bile acids (BA) were determined by standard enzymatic-colorimetric assays using bile-acid assay kits in accordance with the manufacturer's protocols (Wako Pure Chemical Industries).

Determination of hepatic lipids and bile acids. Liver lipid content was extracted according to the method of previous report. ${ }^{(21)}$ Hepatic triglycerides and cholesterol levels were determined enzymatically using commercially available kits (Wako Pure Chemical Industries). Hepatic bile acid concentrations were quantified as described previously. ${ }^{(22)}$

Histopathology. Liver samples were fixed in $10 \%$ formalin prior to routine processing and paraffin embedding. Liver sections ( $4 \mu \mathrm{m}$ in thickness) were stained with hematoxylin and eosin (H\&E) or van Gieson to evaluate severity of histological changes, including steatosis, inflammation, and fibrosis. Histological findings were scored by using "NAFLD scoring system for rodent model".(23)
RNA isolation and real-time polymerase chain reaction. Total RNA was isolated using TRIzol reagent according to the manufacturer's protocol (Life Technologies, Tokyo, Japan). The concentration of total RNA in each sample was quantified spectrophotometrically at $260 \mathrm{~nm}$. The mRNA levels were quantified by SYBR real-time polymerase chain reaction (PCR). The primers are listed in Table 2.(24-26) The amplification reactions were carried out in an ABI Prism 7900 sequence detection system (Applied Biosystems, Foster City, CA). The amount of mRNA was calculated using the comparative CT method which determines the amount of target normalized to an endogenous reference. Each gene was normalized to $18 \mathrm{~s}$ rRNA.

Statistical analysis. The software package, SYSTAT, ver. 11 (Systat Inc., Evanston, IL) was used for statistical analysis. To compare the differences among multiple groups, statistical analysis included analysis of variance, followed by Tukey's multiple range test. Differences were considered statistically significant at $p<0.05$. All the experimental data were expressed as mean $\pm \mathrm{SE}$.

\section{Results}

Effect of oltipraz on hepatic mRNA expression of lipid and bile acids metabolism related genes in mice with standard diet. OPZ is known to be Nrf2 activator, and we investigated the extent to which oral administration of OPZ under this experimental condition altered the expression of the gene products related to bile acid metabolism and lipid metabolism (Table 3 ). With respect to the Nrf2 target gene expression related to bile acid metabolism, Mrp2, Mrp3, small heterodimer partner (Shp), and sterol 12 $\alpha$-hydroxylase (Cyp8b1) were induced in wild type mice, and not in Nrf2-null mice. OPZ induced hepatic mRNA expression of peroxisome proliferator-activated receptor $\alpha$ (Ppar $\alpha$ ) in both wild-type and Nrf2-null mice. Hepatic expression of carnitine palmityl transferase $1(\mathrm{Cpt} 1)$ tended to increase in wild type mice treated with OPZ but not significant, on the other hand, Cpt1 was significantly increased in Nrf2-null mice. With respect to the genes related to bile acid metabolism, hepatic gene expression of Bsep was not significantly increased in wild-type mice treated with OPZ, however, it was significantly increased in Nrf2-null mice by OPZ administration.

Effect of oltipraz on the experimental steatohepatitis in HFD fed Nrf2-null mice.

Serum biochemical markers. Serum total cholesterol, HDL cholesterol, and triglycerides were lower in Nrf2-null mice than in wild-type mice. With respect to liver function test, alkaline phosphatase, alanine aminotransferase, and total bilirubin, tended to be higher in Nrf2-null mice than in wild-type mice. Serum bile 
Table 2. Real-time PCR primer sequences

\begin{tabular}{lll}
\hline Gene symbol & Forward 5'-3' $^{\prime}$ & Reverse 5'-3' $^{\prime}$ \\
\hline Ppar $\alpha$ & TATTCGGCTGAAGCTGGTGTAC & CTGGCATTTGTTCCGGTTCT \\
Acox & GCCCAACTGTGACTTCCATT & GGCATGTAACCCGTAGCACT \\
Cpt1 & GCACTGCAGCTCGCACATTACAA & CTCAGACAGTACCTCCTTCAGGAAA \\
Ppar $\gamma$ & CAGGCTTGCTGAACGTGAAG & GGAGCACCTTGGCGAACA \\
Cd36 & AATGGCACAGACGCAGCCT & GGTTGTCTGGATTCTGGA \\
Srebp1c & GTTACTCGAGCCTGCCTTCAGG & CAAGCTTTGGACCTGGGTGG \\
Fas & GCTGCGGAAACTTCAGGAAAT & AGAGACGTGTCACTCCTGGACTT \\
Lxr $\alpha$ & TCAGCATCTTCTCTGCAGACCGG & TCATTAGCATCCGTGGGAACA \\
Abcg5 & TGGATCCAACACCTCTATGCTAAA & GGCAGGTTTTCTCGATGAACTG \\
Abcg8 & TGCCCACCTTCCACATGTC & ATGAAGCCGGCAGTAAGGTAGA \\
Cyp7a1 & GCTGTGGTAGTGAGCTGTTGCA & CACAGCCCAGGTATGGAATCA \\
Cyp7b1 & CGAGAAGTGCAGGAGGATATGA & GTGGAGGAAAGAGGGCTACAAA \\
Cyp8b1 & GGACTTCCAGTCCTATGCAAAAGA & TGGTACCCAAACACCTTGAGC \\
Oatp1a1 1 & CAGATAAATGGATTTGCCAG & GTCAACAAATAGTTACAGAG \\
Shp & CGATCCTCTTCAACCCAGATG & AGGGCTCCAAGACTTCACACA \\
Bsep & GTTCAGTTCCTCCGTTCAAA & AAGCTGCACTGTCTTTTCAC \\
Mrp2 & CATAAAACCAGGCCAACTGGTG & TGCCCATGGACATTCTCCAT \\
Mrp3 & CAACTTCCTCCGAAACTACGCA & TGCTGAGTGTGTCTTCGAGCAG \\
Nq01 & AGAGTGGCATCCTGCGTTTCT & TTCCATCCTTCCAGGATCTGC \\
Gclc & GCACGGCATCCTCCAGTTCCT & TCGGATGGTTGGGGTTTGCC \\
Gclm & GGCTTCGCCTCCGATTGAAGA & TCACACAGCAGGAGGCCAGGT \\
Tnf $\alpha$ & GACCCTCACACTCAGATCATCTTCT & CCTCCACTTGGTGGTTTGCT \\
Mcp-1 & ACTGAAGCCAGCTCTCTCTTCCTC & TTCCTTCTTGGGGTCAGCACAGAC \\
Timp-1 & ACTCGGACCTGGTCATAAGGGC & TTCCGTGGCAGGCAAGCAAAGT \\
Collagen type I 1 & GCAATTATTCCCCATGAACA & CATCGGTCATGCTCTCTCCAA \\
185 rRNA & GGCCTCACTAAACCATCCAA \\
\hline
\end{tabular}

Table 3. Effect of oltipraz on hepatic mRNA expression of lipid and bile acids metabolism related genes in mice with standard diet

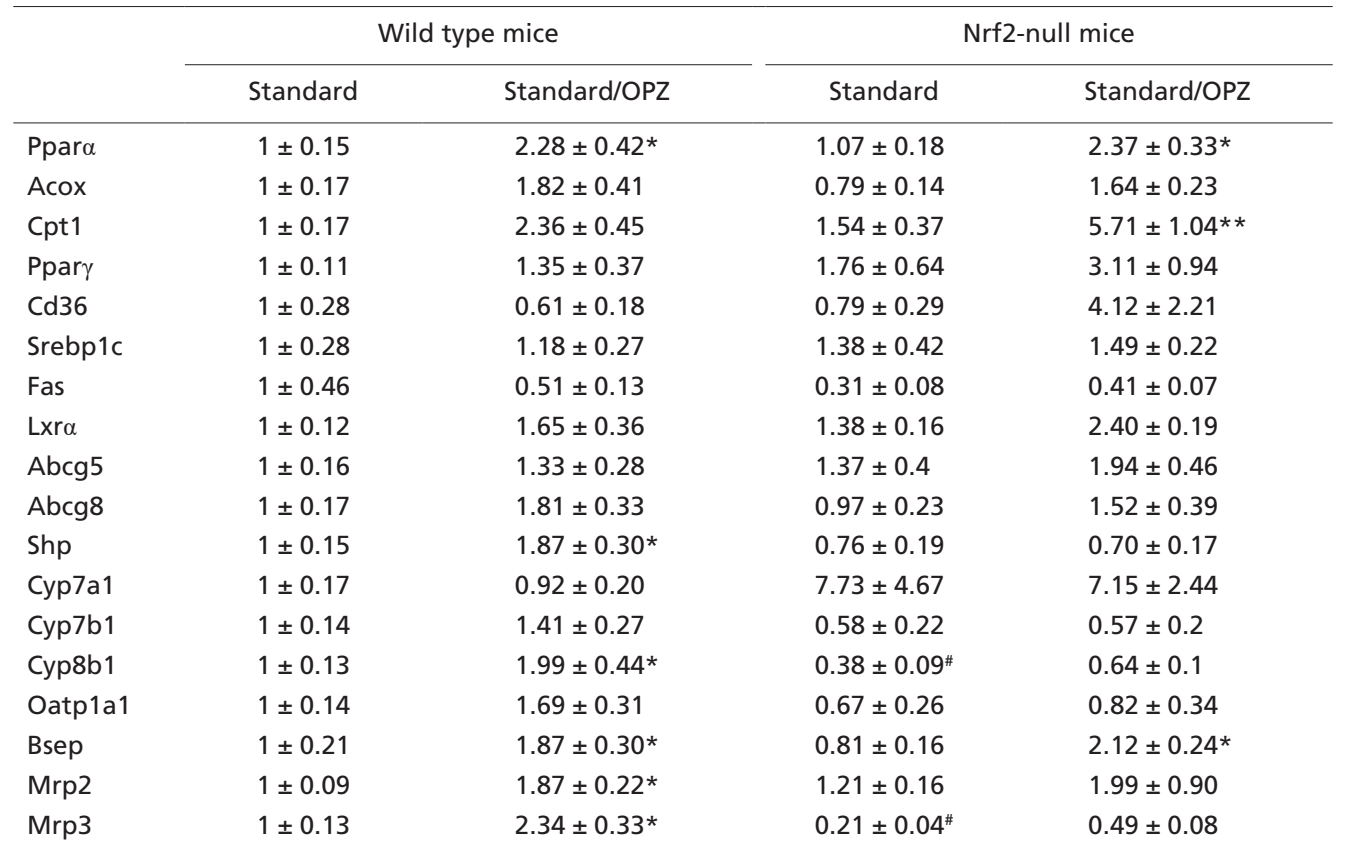

The Data are presented as mean $\pm \mathrm{SE}$. OPZ, oltipraz. ${ }^{*} p<0.05, * * p<0.01$, statistically significant differences compared to the control group within each genotype; ${ }^{*} p<0.05$, statistically significant differences compared to wild-type mice within the same diet group. 
Table 4. Serum biochemical markers, hepatic lipid profiles and hepatic bile acids

\begin{tabular}{|c|c|c|c|c|}
\hline & \multicolumn{2}{|c|}{ Wild type mice } & \multicolumn{2}{|c|}{ Nrf2-null mice } \\
\hline & HFD & $\mathrm{HFD} / \mathrm{OPZ}$ & HFD & $\mathrm{HFD} / \mathrm{OPZ}$ \\
\hline \multicolumn{5}{|l|}{ Serum } \\
\hline Total cholesterol (mg/dl) & $169 \pm 11$ & $134 \pm 21$ & $76 \pm 11^{\#}$ & $106 \pm 17$ \\
\hline Triglycerides (mg/dl) & $24 \pm 11$ & $19 \pm 4$ & $11 \pm 2^{\#}$ & $18 \pm 3$ \\
\hline HDL cholesterol (mg/dl) & $90 \pm 2$ & $74 \pm 11$ & $45 \pm 7^{\#}$ & $61 \pm 8$ \\
\hline ALT (IU/L) & $33 \pm 5$ & $39 \pm 7$ & $64 \pm 20$ & $56 \pm 14$ \\
\hline ALP (IU/L) & $173 \pm 7$ & $193 \pm 27$ & $372 \pm 73$ & $224 \pm 28$ \\
\hline Bilirubin (mg/dl) & $0.02 \pm 0.02$ & $0.05 \pm 0.03$ & $0.18 \pm 0.05$ & $0.13 \pm 0.03$ \\
\hline Bile acids $(\mu \mathrm{mol} / \mathrm{L})$ & $3.96 \pm 1.51$ & $8.16 \pm 1.09$ & $45.85 \pm 16.51^{\#}$ & $13.93 \pm 5.32$ \\
\hline \multicolumn{5}{|l|}{ Liver } \\
\hline Cholesterol (mg/g liver) & $5.94 \pm 0.29$ & $7.34 \pm 0.51$ & $7.48 \pm 0.75$ & $5.87 \pm 0.17$ \\
\hline Triglycerides (mg/g liver) & $152.5 \pm 33.3$ & $147 \pm 25.6$ & $197.4 \pm 54.7$ & $117.4 \pm 28.7$ \\
\hline Bile acids ( $\mu \mathrm{mol} / \mathrm{g}$ liver) & $0.11 \pm 0.03$ & $0.1 \pm 0.04$ & $0.11 \pm 0.01$ & $0.07 \pm 0.01$ * \\
\hline
\end{tabular}

The Data are presented as mean \pm SE. HFD, high fat diet; OPZ, oltipraz. * $p<0.05$, statistically significant differences compared to the control group within each genotype; $\# p<0.05$, statistically significant differences compared to wild-type mice within the same diet group.

Table 5. Effect of oltipraz on hepatic mRNA expression of lipid and bile acids metabolism related genes in mice with high fat diet

\begin{tabular}{|c|c|c|c|c|}
\hline & \multicolumn{2}{|c|}{ Wild type mice } & \multicolumn{2}{|c|}{ Nrf2-null mice } \\
\hline & HFD & HFD/OPZ & HFD & HFD/OPZ \\
\hline Ppar $\alpha$ & $1 \pm 0.12$ & $1.93 \pm 0.39$ & $1.34 \pm 0.16$ & $2.53 \pm 0.39$ * \\
\hline Acox & $1 \pm 0.21$ & $1.27 \pm 0.35$ & $1.21 \pm 0.21$ & $2.04 \pm 0.29 *$ \\
\hline Cpt1 & $1 \pm 0.12$ & $1.71 \pm 0.28$ & $1.45 \pm 0.1$ & $2.52 \pm 0.43^{*}$ \\
\hline Ppar $\gamma$ & $1 \pm 0.22$ & $1.62 \pm 0.44$ & $1.30 \pm 0.39$ & $1.30 \pm 0.17$ \\
\hline $\mathrm{Cd} 36$ & $1 \pm 0.49$ & $0.94 \pm 0.37$ & $0.80 \pm 0.19$ & $0.99 \pm 0.17$ \\
\hline Srebp1c & $1 \pm 0.21$ & $1.84 \pm 0.68$ & $1.08 \pm 0.22$ & $1.07 \pm 0.14$ \\
\hline Fas & $1 \pm 0.22$ & $1.23 \pm 0.37$ & $1.32 \pm 0.30$ & $1.49 \pm 0.21$ \\
\hline Lxr $\alpha$ & $1 \pm 0.10$ & $1.81 \pm 0.24$ & $1.79 \pm 0.22$ & $2.37 \pm 0.29$ \\
\hline Abcg5 & $1 \pm 0.31$ & $1.04 \pm 0.18$ & $0.91 \pm 0.28$ & $1.27 \pm 0.24$ \\
\hline Abcg 8 & $1 \pm 0.23$ & $1.25 \pm 0.26$ & $0.97 \pm 0.32$ & $1.42 \pm 0.10$ \\
\hline Cyp7a1 & $1 \pm 0.22$ & $1.73 \pm 0.38$ & $4.31 \pm 1.73$ & $4.27 \pm 1.30$ \\
\hline Cyp7b1 & $1 \pm 0.27$ & $0.82 \pm 0.17$ & $0.73 \pm 0.29$ & $1.19 \pm 0.21$ \\
\hline Cyp8b1 & $1 \pm 0.18$ & $1.06 \pm 0.18$ & $0.45 \pm 0.10$ & $0.77 \pm 0.12$ \\
\hline Oatp1a1 & $1 \pm 0.15$ & $1.54 \pm 0.23$ & $1.53 \pm 0.34$ & $2.35 \pm 0.27$ \\
\hline Shp & $1 \pm 0.11$ & $1.13 \pm 0.29$ & $0.71 \pm 0.12$ & $1.21 \pm 0.20$ \\
\hline Bsep & $1 \pm 0.16$ & $1.79 \pm 0.2$ & $1.52 \pm 0.25$ & $1.94 \pm 0.21$ \\
\hline Mrp2 & $1 \pm 0.11$ & $1.35 \pm 0.12$ & $1.18 \pm 0.12$ & $1.56 \pm 0.18$ \\
\hline Mrp3 & $1 \pm 0.11$ & $1.36 \pm 0.17$ & $0.26 \pm 0.04^{\#}$ & $0.33 \pm 0.06$ \\
\hline Nqo1 & $1 \pm 0.26$ & $1.34 \pm 0.21$ & $0.29 \pm 0.07^{\#}$ & $0.36 \pm 0.04^{\#}$ \\
\hline Gclc & $1 \pm 0.12$ & $1.38 \pm 0.11$ & $0.74 \pm 0.10$ & $0.96 \pm 0.12$ \\
\hline Gclm & $1 \pm 0.15$ & $1.40 \pm 0.14$ & $0.94 \pm 0.19$ & $1.32 \pm 0.16$ \\
\hline
\end{tabular}

The Data are presented as mean \pm SE. HFD, high fat diet; OPZ, oltipraz. ${ }^{*} p<0.05$, statistically significant differences compared to the control group within each genotype; ${ }^{*} p<0.05$, statistically significant differences compared to wild-type mice within the same diet group.

acid was significantly higher in Nrf2-null mice than in wild-type mice. Serum bile acid concentration was tended to decrease in Nrf2-null mice compared with wild-type mice, but not significant (Table 4).

Hepatic lipid profiles and bile acids. There was no difference in hepatic concentration of cholesterol and triglyceride among the four groups. In Nrf2-null mice, OPZ reduced hepatic bile acid concentrations (Table 4).
Hepatic gene expression of lipid, bile acids metabolism and detoxification related genes. OPZ induced the gene expression of Ppara and its target genes, Acox and Cpt1 in Nrf2-null mice, but not in wild-type mice. With respect to the Nrf2 target gene expression related to bile acid metabolism, Mrp3, Shp, and Cyp8b1 were not induced in either wild-type or Nrf2-null mice. OPZ did not induce the gene expression of Nqo1, Gclc and Gclm both in either wild-type or Nrf2-null mice (Table 5). 
Table 6. Degree of steatosis, inflammation, and fibrosis of liver in wild type and Nrf2-null mice with high fat diet

\begin{tabular}{|c|c|c|c|c|c|}
\hline & & \multicolumn{2}{|c|}{ Wild type mice } & \multicolumn{2}{|c|}{ Nrf2-null mice } \\
\hline & & HFD & HFD/OPZ & HFD & $\mathrm{HFD} / \mathrm{OPZ}$ \\
\hline & (Score) & \multicolumn{4}{|c|}{ Mean of the score } \\
\hline Steatosis & $(0-9)$ & 2.5 & 2.5 & 4.2 & 2.5 \\
\hline Inflammation & $(0-3)$ & 0 & 0.2 & 1.7 & 0.8 \\
\hline \multirow[t]{4}{*}{ Fibrosis } & (Degree) & $(n=6)$ & $(n=6)$ & $(n=6)$ & $(n=6)$ \\
\hline & No & 6 & 5 & 0 & 3 \\
\hline & Mild & 0 & 1 & 5 & 3 \\
\hline & Moderate & 0 & 0 & 1 & 0 \\
\hline
\end{tabular}

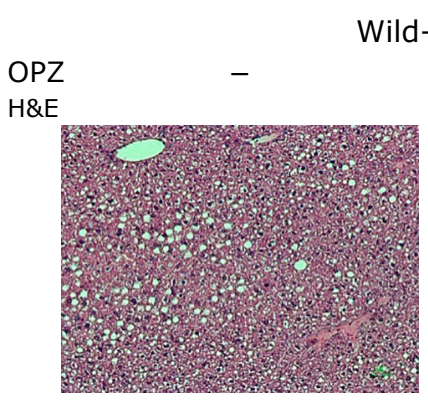

Wild-type

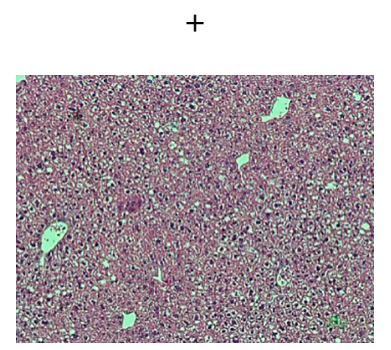

van Gieson

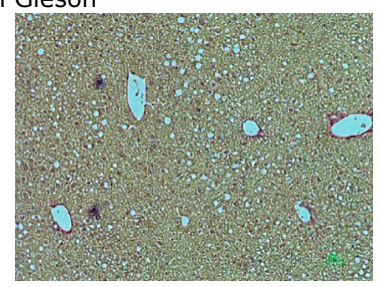

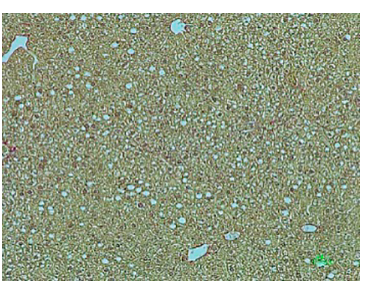
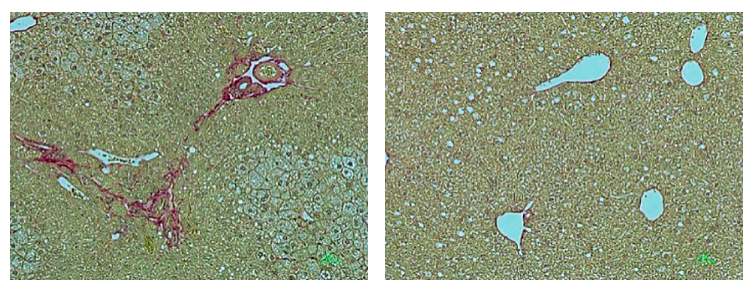

Fig. 1. H\&E and van Gieson staining in wild-type and Nrf2-null mouse liver. Representative photomicrographs of H\&E-stained and van Giesonstained liver sections from wild-type and Nrf2-null mice after 12 weeks of each diet. Representative images are shown.

Table 7. Hepatic gene expression of proinflammatory mediators and fibrogenesis markers in wild-type and Nrf2-null mice

\begin{tabular}{lccccc}
\hline & \multicolumn{2}{c}{ Wild type mice } & \multicolumn{2}{c}{ Nrf2-null mice } \\
\cline { 2 - 3 } \cline { 5 - 6 } & HFD & HFD/OPZ & & HFD & HFD/OPZ \\
\hline Tnf $\alpha$ & $1 \pm 0.27$ & $1.78 \pm 0.45$ & & $5.04 \pm 1.11^{\# \#}$ & $3.34 \pm 1.48$ \\
Mcp-1 & $1 \pm 0.12$ & $2.92 \pm 0.78$ & & $18.46 \pm 3.50^{\# \#}$ & $7.83 \pm 1.61^{* *}$ \\
Timp-1 & $1 \pm 0.28$ & $1.96 \pm 0.6$ & & $30.3 \pm 9.47^{\#}$ & $17.7 \pm 8.31$ \\
Collagen type $1 \alpha 1$ & $1 \pm 0.19$ & $1.06 \pm 0.36$ & & $5.10 \pm 2.23$ & $2.75 \pm 0.69$ \\
\hline
\end{tabular}

The Data are presented as mean \pm SE. HFD, high fat diet; OPZ, oltipraz. ${ }^{*} p<0.01$, statistically significant differences compared to the control group within each genotype; ${ }^{\# p} p<0.05,{ }^{\# \#} p<0.01$, statistically significant differences compared to wild-type mice within the same diet group.

Histology findings of the liver. In wild-type mice, both HFD and HFD/OPZ groups demonstrated moderate to severe hepatic steatosis, however there were no inflammation or fibrosis. On the other hand, in Nrf2-null mice, moderate to severe inflammation and fibrosis were observed in the HFD group. In Nrf2-null mice, the degree of inflammation and fibrosis was milder in the HFD/ OPZ group compared with the HFD group (Table 6 and Fig. 1).

Hepatic gene expression of proinflammatory mediators and fibrogenesis markers. Hepatic expression of tumor necrosis factor $\alpha(\mathrm{TNF} \alpha)$, monocyte chemoattractant protein-1 (Mcp-1), tissue inhibitor of metalloproteinase-1 (Timp-1), and collagen type $I \alpha 1$, reflecting the degree of liver inflammation and fibrosis, was not changed by OPZ administration in wild-type mice. The expression of Tnfa, Mcp-1, and Timp-1 were higher in Nrf2-null mice without OPZ than in wild-type mice without OPZ. In Nrf2null mice, the gene expression of $\mathrm{Mcp}-1$ was significantly decreased by OPZ administration. Hepatic expression of Tnfa, Timp-1, and collagen type $1 \alpha 1$ tended to be attenuated by OPZ in Nrf2-null mice, but these changes were not statistically significant (Table 7). 


\section{Discussion}

Because the administration of Nrf2 activators ameliorates hepatic lipid accumulation and hepatic fibrosis, ${ }^{(12-15)}$ and because Nrf2 deficiency develops steatohepatitis, ${ }^{(11,12)}$ it is thought that Nrf2 is one of the key molecules for the development of steatohepatitis in rodents. This phenomenon is thought to be related to the expression of several detoxifying and antioxidant enzymes that are regulated by Nrf2, ${ }^{(27,28)}$ as well as the regulatory effect of Nrf2 on lipid and bile acid metabolism. ${ }^{(3-7,16,29)}$ OPZ has been reported to attenuate hepatic fibrosis in experimental rodent models of steatohepatitis. ${ }^{(17)}$ Therefore, the pharmacological effect of OPZ on this experimental steatohepatitis was thought to be Nrf2-dependent. On the other hand, OPZ has also been reported to have Nrf2-independent pharmacological effect. ${ }^{(16,18)}$ In this study, we demonstrated that OPZ had the Nrf2 independent effect on lipid and bile acid metabolism, and that OPZ ameliorated the experimental steatohepatitis caused by 12 weeks of HFD treatment in Nrf2-null mice. This finding suggests that the pharmacological action of OPZ for this experimental steatohepatitis is through Nrf2 independent manner.

Under condition fed a standard diet containing 4\% soybean oil, hepatic expression of Nrf2 target genes such as Mrp2, Mrp3, Shp, and Cyp8b1 was increased by OPZ in wild-type mice, but not in Nrf2-null mice. Therefore, the oral administration dose and the duration of OPZ in this study was thought to be an appropriate level as Nrf2 activator. On a standard diet, hepatic expression of fatty acid oxidation related gene, including Ppar $\alpha$, and Cpt1 and bile acid transporters, including Bsep was induced by OPZ in Nrf2-null mice. These results suggest that OPZ has the effects on regulation of the gene product related to lipid and bile acid metabolism not only through Nrf2 dependent pathway but also through Nrf2 independent pathway. On the other hand, under condition fed a high fat diet, there were little induction of Nrf2 target gene by OPZ. These results suggest that OPZ is no longer able to further induce the Nrf2 pathway in HFD condition.

Several previous studies have shown that targeted deletion of Nrf2 leads to enhanced susceptibility to hepatic injury, and rapid progression of steatohepatitis in Nrf2-null mice with high-fat diet. $^{(12)}$ In the present study, after 12 weeks of HFD feeding, wild-type mice showed fatty deposits, but no inflammation or fibrosis in the liver. On the other hand, in Nrf2-null mice fed a HFD for 12 weeks, hepatic fat deposits as well as inflammation and fibrosis were observed in the liver as previously reported.(12) Increase in pro-inflammatory cytokines such as TNF $\alpha$ as well as dysregulation of antioxidative system is thought to be related to the susceptibility of Nrf2-null mice to steatohepatitis in HFD. ${ }^{(12)}$ In addition to these mechanisms, the hepatic damages are thought to be caused by dysregulation of fatty acid and bile acid metabolism in the absence of Nrf2. However, in this study, there was no difference in hepatic lipid concentrations and hepatic expression of fatty acid metabolism related gene product between HFD-fed wild-type and Nrf2-null mice. Regarding bile acid metabolism, Cyp7a1 expression tended to be higher and Mrp3 expression was lower in Nrf2-null mice, but there was no significant difference in hepatic bile acid concentration between wildtype and Nrf2-null mice. Because of these results, we could not

\section{References}

1 Ishii $\mathrm{T}$, Itoh $\mathrm{K}$, Yamamoto $\mathrm{M}$. Roles of Nrf2 in activation of antioxidant enzyme genes via antioxidant responsive elements. Methods Enzymol 2002; 348: $182-190$

2 Maher JM, Cheng X, Slitt AL, Dieter MZ, Klaassen CD. Induction of the multidrug resistance-associated protein family of transporters by chemical activators of receptor-mediated pathways in mouse liver. Drug Metab Dispos 2005; 33: 956-962. conclude that the steatohepatitis in Nrf2-null mice fed a HFD is due to disturbance of fatty acid or bile acid metabolism caused by deficiency of Nrf2.

Number of studies demonstrated that Nrf2 activators have been shown to inhibit liver fibrosis in rodent models of steatohepatitis. ${ }^{(13-15,17)}$ These findings suggest that Nrf2 activation may be a target for the treatment of steatohepatitis. In the present study, OPZ reduced fat deposition and the expression of genes associated with inflammation and fibrosis, including Tnfo, Mcp-1, Timp-1 and collagen type 1a1 in Nrf2-null mouse liver with HFD feeding. These findings suggest that OPZ may inhibit fat deposit, inflammation, and fibrosis by $\mathrm{Nrf} 2$ independent mechanisms. Although the mechanism underlying the Nrf2independent pharmacological effect of OPZ observed in this study is not clear, we demonstrated that OPZ induced the expression of Ppara and its target gene Cpt1 in Nrf2-null mice. Ppara has a key role in regulation of fatty acid $\beta$-oxidation and it has been reported that HFD induced $\beta$-oxidation by Nrf2 independent mechanisms. ${ }^{(12)}$ These findings suggest that OPZ may further activate fatty acid $\beta$-oxidation even in the presence of HFDinduced activation of PPAR $\alpha$ pathway in Nrf2 null mice. In addition to the role in fatty acid $\beta$ oxidation, Ppar $\alpha$ suppresses inflammatory cytokines by competitively inhibiting nuclear factorkappa B (NF-kappa B), a master regulator of inflammation. ${ }^{(30)}$ In fact, Ppar $\alpha$ is known to protect the liver from HFD-induced steatosis and inflammation. ${ }^{31,32)}$ Furthermore, it has been reported that pemafibrate, a selective PPAR $\alpha$ modulator, improves hepatic steatosis, inflammation, and fibrosis in rodent experimental steatohepatitis model. ${ }^{(33)}$ PPAR $\alpha$ is considered to have a protective role against the pathogenesis of steatohepatitis. Therefore, the activation of Ppara is one of the candidate mechanisms for Nrf2-independent hepatoprotective effect of OPZ against steatohepatitis in Nrf2-null mice fed a HFD. OPZ is also known as a CAR activator. ${ }^{(18)}$ With respect to the relation between PPAR $\alpha$ and CAR, it has been reported that PPAR $\alpha$ agonist induced the drive of CAR into hepatocyte nuclei. ${ }^{(34)}$ Furthermore, PPAR $\alpha$-null mice showed enhanced hepatocyte proliferation in response to CAR agonist. ${ }^{(35)}$ These findings suggest that there may be some functional relation between PPAR $\alpha$ and CAR.

In conclusion, this study showed that OPZ administration ameliorated steatohepatitis developed in Nrf2-null mice when placed on a HFD in an Nrf2-independent manner. Therefore, OPZ has hepatoprotective effects via Nrf2 dependent and independent mechanisms.

\section{Acknowledgments}

This work was supported by JSPS KAKENHI (Grants 23580188 and 24501010). The authors would like to thank Dr. Masayuki Yamamoto for providing Nrf2-null breeding pairs and Mr. Yasumitsu Akahoshi, Ms. Yoko Saito, and Ms. Mariko Shibayama for technical assistance.

\section{Conflict of Interest}

No potential conflicts of interest were disclosed.
3 Tanaka Y, Aleksunes LM, Yeager RL, et al. NF-E2-related factor 2 inhibits lipid accumulation and oxidative stress in mice fed a high-fat diet. $J$ Pharmacol Exp Ther 2008; 325: 655-664.

4 Zhang YK, Yeager RL, Tanaka Y, Klaassen CD. Enhanced expression of Nrf2 in mice attenuates the fatty liver produced by a methionine- and cholinedeficient diet. Toxicol Appl Pharmacol 2010; 245: 326-334.

5 Tan KP, Wood GA, Yang M, Ito S. Participation of nuclear factor (erythroid 
2-related), factor 2 in ameliorating lithocholic acid-induced cholestatic liver injury in mice. Br J Pharmacol 2010; 161: 1111-1121.

6 Weerachayaphorn J, Cai SY, Soroka CJ, Boyer JL. Nuclear factor erythroid 2related factor 2 is a positive regulator of human bile salt export pump expression. Hepatology 2009; 50: 1588-1596.

7 Xu S, Weerachayaphorn J, Cai SY, Soroka CJ, Boyer JL. Aryl hydrocarbon receptor and NF-E2-related factor 2 are key regulators of human MRP4 expression. Am J Physiol Gastrointest Liver Physiol 2010; 299: G126-G135.

8 Kamisako T, Tanaka Y, Kishino Y, et al. Role of Nrf2 in the alteration of cholesterol and bile acid metabolism-related gene expression by dietary cholesterol in high fat-fed mice. J Clin Biochem Nutr 2014; 54: 90-94.

9 Ramos-Gomez M, Kwak MK, Dolan PM, et al. Sensitivity to carcinogenesis is increased and chemoprotective efficacy of enzyme inducers is lost in nrf2 transcription factor-deficient mice. Proc Natl Acad Sci U S A 2001; 98: 3410 3415 .

10 Maher JM, Dieter MZ, Aleksunes LM, et al. Oxidative and electrophilic stress induces multidrug resistance-associated protein transporters via the nuclear factor-E2-related factor-2 transcriptional pathway. Hepatology 2007; 46: $1597-1610$.

11 Sugimoto H, Okada K, Shoda J, et al. Deletion of nuclear factor-E2-related factor-2 leads to rapid onset and progression of nutritional steatohepatitis in mice. Am J Physiol Gastrointest Liver Physiol 2010; 298: G283-G294.

12 Okada K, Warabi E, Sugimoto H, et al. Deletion of Nrf2 leads to rapid progression of steatohepatitis in mice fed atherogenic plus high-fat diet. $J$ Gastroenterol 2013; 48: 620-632.

13 Shin S, Wakabayashi J, Yates MS, et al. Role of Nrf2 in prevention of highfat diet-induced obesity by synthetic triterpenoid CDDO-imidazolide. Eur $J$ Pharmacol 2009; 620: 138-144.

14 Saha PK, Reddy VT, Konopleva M, Andreeff M, Chan L. The triterpenoid 2cyano-3,12-dioxooleana-1,9-dien-28-oic-acid methyl ester has potent antidiabetic effects in diet-induced diabetic mice and Lepr(db/db) mice. $J$ Biol Chem 2010; 285: 40581-40592.

15 Oh CJ, Kim JY, Min AK, et al. Sulforaphane attenuates hepatic fibrosis via NF-E2-related factor 2-mediated inhibition of transforming growth factor- $\beta$ / Smad signaling. Free Radic Biol Med 2012; 52: 671-682.

16 Tanaka Y, Aleksunes LM, Cui YJ, Klaassen CD. ANIT-induced intrahepatic cholestasis alters hepatobiliary transporter expression via Nrf2-dependent and independent signaling. Toxicol Sci 2009; 108: 247-257.

17 Shimozono R, Asaoka Y, Yoshizawa Y, et al. Nrf2 activators attenuate the progression of nonalcoholic steatohepatitis-related fibrosis in a dietary rat model. Mol Pharmacol 2013; 84: 62-70.

18 Merrell MD, Jackson JP, Augustine LM, et al. The Nrf2 activator oltipraz also activates the constitutive androstane receptor. Drug Metab Dispos 2008; 36: 1716-1721.

19 Bae EJ, Yang YM, Kim JW, Kim SG. Identification of a novel class of dithiolethiones that prevent hepatic insulin resistance via the adenosine monophosphate-activated protein kinase-p70 ribosomal S6 kinase-1 pathway. Hepatology 2007; 46: 730-739.

20 Hwahng SH, Ki SH, Bae EJ, Kim HE, Kim SG. Role of adenosine monophosphate-activated protein kinase-p70 ribosomal S6 kinase-1 pathway in repression of liver $\mathrm{X}$ receptor-alpha-dependent lipogenic gene induction and hepatic steatosis by a novel class of dithiolethiones. Hepatology 2009; 49: 1913-1925.
21 Folch J, Lee M, Sloane Stanley GH. A simple method for the isolation and purification of total lipids from animal tissues. J Biol Chem 1957; 226: $497-$ 509.

22 Bohan A, Chen WS, Denson LA, Held MA, Boyer JL. Tumor necrosis factor alpha-dependent up-regulation of Lrh-1 and Mrp3 (Abcc3) reduces liver injury in obstructive cholestasis. J Biol Chem 2003; 278: 36688-36698.

23 Liang W, Menke AL, Driessen A, et al. Establishment of a general NAFLD scoring system for rodent models and comparison to human liver pathology. PLoS One 2014; 9: e115922.

24 Kamisako T, Tanaka Y, Ikeda T, Yamamoto K, Ogawa H. Dietary fish oil regulates gene expression of cholesterol and bile acid transporters in mice. Hepatol Res 2012; 42: 321-326.

25 Kishino Y, Tanaka Y, Ikeda T, et al. Ezetimibe increases hepatic iron levels in mice fed a high-fat diet. $J$ Pharmacol Exp Ther 2013; 345: 483-491.

26 Tanaka Y, Ikeda T, Yamamoto K, Ogawa H, Kamisako T. Dysregulated expression of fatty acid oxidation enzymes and iron-regulatory genes in livers of Nrf2-null mice. J Gastroenterol Hepatol 2012; 27:1711-1717.

27 Braun S, Hanselmann C, Gassmann MG, et al. Nrf2 transcription factor, a novel target of keratinocyte growth factor action which regulates gene expression and inflammation in the healing skin wound. Mol Cell Biol 2002; 22: 5492-5505.

28 Iida K, Itoh K, Kumagai Y, et al. Nrf2 is essential for the chemopreventive efficacy of oltipraz against urinary bladder carcinogenesis. Cancer Res 2004; 64: 6424-6431.

29 Weerachayaphorn J, Mennone A, Soroka CJ, et al. Nuclear factor-E2-related factor 2 is a major determinant of bile acid homeostasis in the liver and intestine. Am J Physiol Gastrointest Liver Physiol 2012; 302: G925-G936.

30 Pineda Torra I, Gervois P, Staels B. Peroxisome proliferator-activated receptor alpha in metabolic disease, inflammation, atherosclerosis and aging. Curr Opin Lipidol 1999; 10: 151-159.

31 Stienstra R, Mandard S, Patsouris D, Maass C, Kersten S, Müller M. Peroxisome proliferator-activated receptor alpha protects against obesity-induced hepatic inflammation. Endocrinology 2007; 148: 2753-2763.

32 Abdelmegeed MA, Yoo SH, Henderson LE, Gonzalez FJ, Woodcroft KJ, Song BJ. PPARalpha expression protects male mice from high fat-induced nonalcoholic fatty liver. J Nutr 2011; 141: 603-610.

33 Honda Y, Kessoku T, Ogawa Y, et al. Pemafibrate, a novel selective peroxisome proliferator-activated receptor alpha modulator, improves the pathogenesis in a rodent model of nonalcoholic steatohepatitis. Sci Rep 2017; 7: 42477.

34 Guo D, Sarkar J, Suino-Powell K, et al. Induction of nuclear translocation of constitutive androstane receptor by peroxisome proliferator-activated receptor alpha synthetic ligands in mouse liver. J Biol Chem 2007; 282: 36766-36776.

35 Columbano A, Ledda-Columbano GM, Pibiri M, Concas D, Reddy JK, Rao MS. Peroxisome proliferator-activated receptor-alpha mice show enhanced hepatocyte proliferation in response to the hepatomitogen 1,4-bis [2-(3,5dichloropyridyloxy)] benzene, a ligand of constitutive androstane receptor Hepatology 2001; 34: 262-266.

This is an open access article distributed under the terms of the Creative Commons Attribution-NonCommercial-NoDerivatives License (http://creativecommons.org/licenses/by-nc-nd/4.0/). 